\title{
Comprehensive Relevance of AMPK in Adaptive Responses of Physical Exercise, Skeletal Muscle and Neuromuscular Disorders
}

\author{
Jun-Ho Lee, $\mathrm{PhD}^{\dagger}$
}

Department of Emergency Medical Technology, College of Health and Medical Science, Daejeon University

Received: July 21, 2018 / Revised: July 282018 / Accepted: August 2, 2018

(C) 2018 J Korean Soc Phys Med

\section{| Abstract |}

PURPOSE: This study was conducted to understand the adaptive responses of different modes of physical exercises utilizing skeletal muscle and the comprehensive relevance of AMPK signaling that can be activated by physical exercise as a potential molecular target in human health problems such as neuromuscular disorders (NMDs).

METHODS: Most of the contents in this review article are based on recent publications concerning the main topics of interest. The reference literatures cited were obtained by basic searches of overseas academic databases such as PubMed and ScienceDirect using EndNote X7.8.

RESULTS: The phenotypic adaptive responses of skeletal muscle during endurance- and resistance-based exercise training (ET and RT respectively) appear to be distinct. To explain the adaptive responses in each single mode of exercises (ET, RT) along with combined exercise training (CT), AMPK signaling is proposed as an important molecular

$\dagger$ Corresponding Author : Jun-Ho Lee

jhlee@dju.kr, http://orcid.org/0000-0003-1572-1225

This is an Open Access article distributed under the terms of the Creative Commons Attribution Non-Commercial License (http://creativecommons.org/licenses/by-nc/3.0) which permits unrestricted non-commercial use, distribution, and reproduction in any medium, provided the original work is properly cited. link among those differential modes of exercise and a promising molecular target of NMDs.

CONCLUSION: Based on the available evidence, intracellular AMPK signaling activated by diverse stimuli including physical exercise can be a potential and promising therapeutic target for the prevention, amelioration or cure of various human health problems including NMDs and may also be beneficial for physical rehabilitation and emergency situations that may elicit acute metabolic stresses.

Key Words: AMPK, Concurrent exercise training (CT), Endurance-based exercise training (ET), Neuromuscular disorders (NMDs), Resistance-based exercise training (RT),

Skeletal muscle

\section{Introduction}

Physical exercise requires coordinated actions of skeletal muscles and its beneficial effects are known to be diverse. Some people perform exercise to maintain healthy physical conditions, lose weight or reinforce physical strength and capacity to achieve a better athletic performance while others exercise to improve or cure health problems such as cardiovascular disease, diabetes or neuromuscular disorders (NMDs). However, depending on the modes of physical exercise, the consequences of physical exercise 
vary. Furthermore, the molecular mechanisms underlying the diversity and dissimilarity in adaptive responses of physical exercise are predominantly unclear and often too complex to fully understand based on currently available information. Nevertheless, it is necessary to identify the characteristics and significance of physical exercise with skeletal muscular activity and to comprehend essential molecular links associated with the beneficial outcomes of physical exercise. In this review, AMPK signaling is predominantly introduced as a major molecular player in this liaison and a promising therapeutic target with molecular mechanistic explanations in NMDs in particular.

Characteristics of Skeletal Muscle and Adaptive Responses in Physical Exercise

The primary task of skeletal muscle is to impart mechanical forces through tendons to bone. Specifically, skeletal muscle provides different amounts of contractile forces for a wide range of movements based on the type, properties and metabolism of corresponding muscles. Some muscles need to sustain contraction for prolonged periods regardless of response latency, while others must respond swiftly for short bursts of activity. Skeletal muscle is characterized by dynamic and flexible adaptability in response to diverse stimuli including physical exercise (Preston and Wilson, 2013; Mounier et al., 2015; Camera et al., 2016).

Physical exercise is frequently divided into two modes: endurance- and resistance-based exercises (primarily aerobic and anaerobic, respectively). The former is known to be more closely related to improving muscular functional capacity to sustain exercise through relatively prolonged $(>60 \mathrm{~min})$ continuous or discontinuous repetition of submaximal muscular contractions demanding endurance, while the latter develops muscular strength to cope with a burden of resistance during a relatively short period of time ( $<60 \mathrm{~s}$ ) with nearly maximal muscular contractions. Muscle fibers vary in composition and function according to fitness requirements assigned (Preston and Wilson, 2013; Mounier et al., 2015; Camera et al., 2016). Depending on the types of stimuli and choice of intensity, frequency and duration, muscle tissues adapt to meet the external demands in the forms of qualitative phenotypic transformation of muscle fibers (primarily in endurance exercise) or hypertrophy with the growth of muscle fibers (mainly in resistance exercise).

The common characteristics of skeletal muscle are also described by two specific types of muscle fibers, type I and type II twitch fibers. Type I slow twitch fibers (red muscle), which dominate muscle, contract slowly and are developed by low-intensity and long-duration activities such as maintaining posture (static isometric exercise) or rhythmic cycles of walking (dynamic isotonic exercise). Type II fast twitch fibers (white muscle) are developed during high-intensity and heavy resistance exercise, such as weight lifting or sighting-pursuing prey eliciting fast fatigue (Preston and Wilson, 2013; Camera et al., 2016). In addition, there are significant differences in oxidative and glycolytic capacities in terms of the predominant energy systems utilized in addition to myoglobin content, mitochondrial size and numbers, myosin ATPase activity as well as resistance to fatigue (Mounier et al., 2015).

Specifically Endurance exercise tends to change skeletal muscle fibers from primarily glycolytic fibers (type IIb/IIx) to more oxidative fibers (type I and IIa) to meet the requirements of energy production though glycolytic pathways are also well-developed in oxidative fibers. Muscular plasticity and adaptation can be achieved in a representative form of skeletal muscular changes in size, composition, and distribution as well as in strength and capacity in response to various stimuli such as specific modes of physical exercise (Preston and Wilson, 2013; Hawley et al., 2014; Mounier et al., 2015).

Furthermore, there is also combined integration of endurance- and resistance-based exercises in a training sequence that is referred to as concurrent training (Coffey 


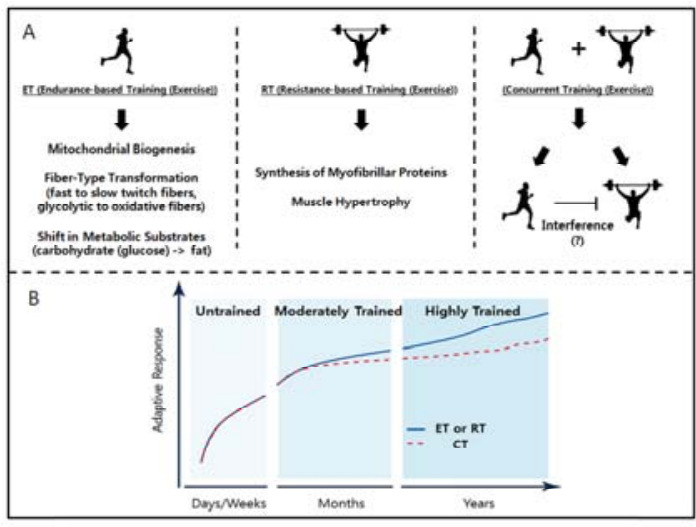

Fig. 1. Comparison of Single Modes of Exercise Training and Concurrent Training. (A) Simplified schematic diagram of phenotypic adaptive responses obtained from single modes of exercise training (endurance-based exercise training (ET) or resistance-based exercise training (RT)) with interrupted adaptive response in concurrent exercise training (CT) is presented. Interference in adaptive response between ET and RT was also indicated in CT although this is still controversial. (B) Hypothetical and speculative time course of adaptive responses in ET, RT and CT from an untrained state to a highly trained state. Interference in adaptive response becomes more evident as training periods are prolonged between single modes of exercise training (ET or RT) and concurrent exercise training (CT) (Adapted from (Coffey and Hawley, 2017)).

and Hawley, 2017). Concurrent training is a sequential exercise regimen and training program where components of endurance-based exercise (aerobic exercise, cardiovascular exercise) and resistance-based exercise (strength exercise) are combined. Numbers of observations demonstrate endurance- and resistance-based exercise in concurrent training result in a compromised adaptive response referred to as interference effect, particularly when resistance training is preceded by endurance-based exercise (Hickson, 1980; Dudley and Djamil, 1985; Chtara et al., 2008; Wilson et al., 2012; Jones et al., 2013; Coffey and Hawley, 2017). However, the precise mechanistic explanation behind this effect is still vague and dissimilar or even contrary evidence also exist (Wang et al., 2011; Murach and Bagley, 2016; Coffey and Hawley, 2017; Murlasits et al., 2018). This controversy might be due in part to complex signaling networks of convergent and divergent signaling pathways under each single mode of exercise training (endurancebased exercise training (ET), resistance-based exercise training (RT)) and/or the existence of undefined essential molecular signaling link(s) between them. Additionally, numerous possible combinations of exercise training paradigms make it more complicated (Fig. 1).

\section{AMPK in Skeletal Muscle and Physical Exercise}

Skeletal muscular plasticity entails metabolic changes with alterations in intracellular signaling pathways. Depending on modes of exercise, cascades of signaling molecules in the corresponding signaling pathway can be activated or suppressed. In terms of signaling pathways to achieve such adaptability and suitability of skeletal muscle, distinct physiological demands need to adopt and regulate specific signaling molecules in the pathway by means of protein activation and posttranslational modifications other than differential gene expression (Bassel-Duby and Olson, 2006).

Among the various signaling molecules in physical exercise, AMP-activated protein kinase (AMPK) has been accredited as a master energy sensor molecule, especially in tissues showing high energy turnover such as skeletal muscle (Richter and Ruderman, 2009; Hawley et al., 2014; Mounier et al., 2015; Camera et al., 2016; Morales-Alamo and Calbet, 2016; Kjobsted et al., 2018). Alterations in energy charge in skeletal muscle induce activation of AMPK, a serine/threonine kinase activated by low cellular energy status signaled from lower levels of ATP, which is a major energy fuel source and/or by higher AMP/ATP (to a lesser extent ADP/ATP) ratios inside the cell. During muscle contraction, the adenylate charge, ATP is decreased and the AMP/ATP ratio is increased, thereby leading to 
AMPK activation (Hardie, 2011a, 2011b).

AMPK is a heterotrimer comprised of two isoforms of $\alpha$ and $\beta$ subunits and three isoforms of $\gamma$ subunit possibly expressing 12 different AMPK complexes. Among the various tissue-specific expressions of AMPK, $a_{2} \beta_{2} \gamma_{1}$ is recognized to be the dominant composition of AMPK in human and rodent skeletal muscle (Mounier et al., 2015; Kjobsted et al., 2018). The allosteric binding of AMP/ADP to the regulatory $\gamma$ subunit of AMPK induces its conformational change and promotes $\mathrm{a}-\mathrm{Thr} 172$ phosphorylation, which is critical for AMPK activation (Stein et al., 2000; Gowans et al., 2013).

Muscle fibers promptly adjust to changes in energy demand during exercise, which is a potent physiological activator of AMPK (Jensen et al., 2009; O'Neill, 2013). During exercise, activated AMPK enhances energyyielding procedures such as glucose uptake and fatty acid oxidation and decreases energy-consuming actions including protein and lipid synthesis. In general, while anaerobic metabolism produces ATP rapidly via ATP-creatine phosphate (CP) and lactic acid systems within a limited duration (seconds), aerobic metabolism generates ATP slowly and sustains activity longer (hours) mostly through oxidative phosphorylation (OXPHOS). AMPK regulates energy homeostasis and metabolism by fine tuning anabolic and catabolic signaling pathways with the control of glycolytic or oxidative phenotype (Mounier et al., 2015; Camera et al., 2016; Kjobsted et al., 2018).

AMPK and AMPK-related Signaling Pathways in Physical Exercise

As previously mentioned, endurance-based exercise upregulates mitochondrial content, biogenesis and respiratory capacity with oxidative and slow twitch fiber-type transformation as well as metabolic shifts such as fat oxidation during submaximal exercise, while resistancebased exercise potentiates synthesis of myofibrillar proteins accountable for muscle hypertrophy and upregulates maximal strength of skeletal muscle (Holloszy, 1967; Holloszy and Coyle, 1984; Tesch, 1988; Fry, 2004; Coffey and Hawley, 2007). However, a single bout of exercise is insufficient to induce morphological changes in each mode of exercise. Rather, it takes time to achieve changes in phenotypic expression via repeated and continuous accumulation of transient alterations (Widegren et al., 2001; Hawley, 2009). Because adaptive responses of each single mode of exercise (ET and RT) appear to be distinct and mutual influences between them seem to be complicated, simple but plausible molecular interpretations are needed to explain the outcomes of single or combined modes of exercise.

One of the best known intracellular signaling pathways mediating protein synthesis in resistance-based exercise is PI3-k (phosphatidylinositol 3-kinase)-Akt-mTOR (mammalian target of rapamycin) cascade (Sakamoto and Goodyear, 2002; Baar, 2006; Deshmukh et al., 2008; Cao et al., 2012; Kwon et al., 2018). Consecutive downstream effectors in this signaling pathway could be p70S6K (ribosomal protein S6 kinase) and 4EBP1 (eIF4E-bindingprotein), which can potentiate translation and protein synthesis, finally contributing to muscle hypertrophy (Hawley, 2009; Richter and Ruderman, 2009; Reidy et al., 2014; Perez-Schindler et al., 2015; Kwon et al., 2018). The molecular basis for the phenotypic manifestation of resistance-based exercise is primarily upregulated translational activity rather than augmented transcription. In contrast, adaptive response of endurance-based exercise appears to show the opposite trend. Potentiated transcriptional activity of genes encoding mitochondrial biogenesis and oxidative phenotypes is likely to be a primary explanation for the adaptive response of endurance-based exercise. The AMPK and its downstream target, PGC-1a (peroxisome proliferator-activated receptor gamma coactivator 1-alpha) appear to be converging signaling molecules in endurance-based exercise that are subsequently followed by activation of various transcription factors including CREB (cAMP response element-binding 


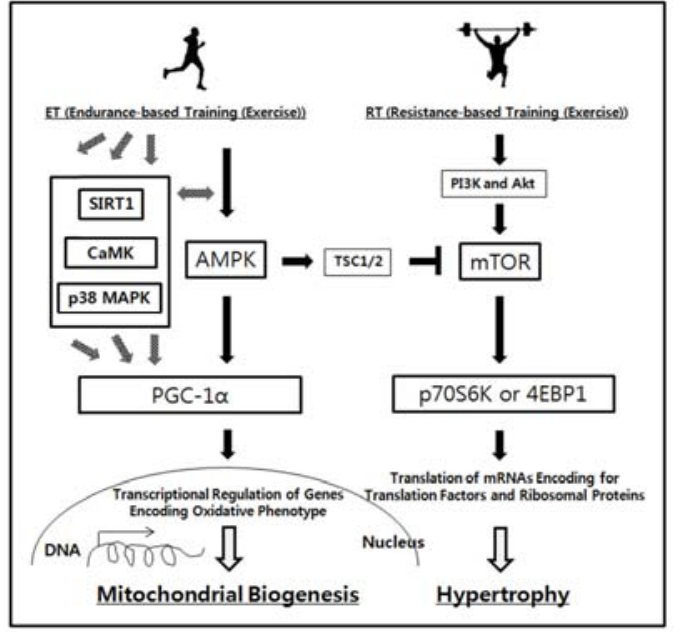

Fig. 2. Simplified Signaling Pathways of AMPK Related to Single Modes of Exercise Training. Representative distinctive signaling pathways of endurance-based exercise training (ET) and resistance-based exercise training (RT) are shown in this diagram. As a molecular signaling link between ET and RT, the inhibition of mTOR by ET-mediated AMPK activation via TSC1/2 is also presented. In addition to AMPK, some other feasible upstream activators of PGC-1 a such as SIRT1, CaMK and p38 MAPK, which may be dependent or independent of AMPK signaling and their mutual influences in ET are also indicated (SIRT1: Sirtuin 1 (NAD(+)-dependent deacetylase sirtuin-1), CaMK: $\mathrm{Ca}^{2+} /$ calmodulindependent protein kinase, p38 MAPK: P38 mitogen-activated protein kinase).

protein) and NRFs (nuclear respiratory factors) via interaction with PPAR- $\gamma$ (peroxisome proliferator-activated receptors). These encode genes for oxidative phenotypes such as mitochondrial biogenesis with an increase in mitochondrial content and density as well as oxidative enzymatic activities (Coffey and Hawley, 2007; Hawley, 2009; Camera et al., 2016; Coffey and Hawley, 2017).

In particular, AMPK is an upstream effector of PGC-1a activation that is able to inhibit mTOR via TSC1 and TSC2 as a source of molecular signaling linkage between endurance- and resistance-based exercises. This connection might be able to explain why interference in concurrent training, if correct, occurs when endurance-based training is followed by resistance training (Fig. 2). However, further investigation on the effects of exercise sequence in concurrent training is required since altered sequences of exercise can produce dissimilar outcomes. For example, when resistance-based training prior to endurance-based training is performed, there seems to be a beneficial effect instead of interference with lower body strength adaptations (Murlasits et al., 2018).

There are many more molecular connections in AMPK signaling including SIRT1, CaMK, p38 MAPK, LKB1 and p53 (Hawley et al., 2006; Gibala, 2009; Hawley, 2009; Perez-Schindler et al., 2015; Beyfuss and Hood, 2018). Especially, p53 is a well-known tumor suppressor whose major regulators are MDM2 (mouse double minute 2) and MDM4 (mouse double minute 4, MDMX) is also reported to influence the regulation of AMPK (Jensen et al., 2007) and to play a significant role in glucose metabolism to regulate glycolysis and oxidative phosphorylation (OXPHOS), which are important to the expression of phenotypes associated with both endurance- and resistancebased exercises (Beyfuss and Hood, 2018; Gomes et al., 2018). Moreover, p53 has a negative influence on metabolic reprogramming, particularly glucose metabolism, is known to revert to the Warburg effect, which is the augmentation of aerobic glycolysis favoring glucose uptake and lactate production, even in the presence of oxygen to generate ATP (Warburg, 1930; Warburg, 1956; Vander Heiden et al., 2009; Vousden and Ryan, 2009; Gomes et al., 2018). p53 decreases glycolytic flux and increases OXPHOS flow by transcriptional or posttranslational regulation of various genes and proteins such as a number of enzymes involved in respective glycolytic and OXPHOS pathways (Gomes et al., 2018). Because of the considerable role of p53 in metabolic regulation, which is important for the outcomes of endurance- and resistance-based exercises, p53-related signaling might be another promising molecular link in 
concurrent exercise training. Hence, the molecular mechanisms and interrelationship of single or combined modes of exercise-based training are likely to be much more complicated and imprecise necessitating much further investigation.

\section{AMPK in Neuromuscular Disorders}

AMPK is a substantial regulator of intracellular metabolism that is essential to neuromuscular determination and maintenance of skeletal muscle, alpha motor neurons (aMNs) and neuromuscular junctions (NMJs) (Dial et al., 2018). Pharmacological or physiological activation of AMPK indicates positive effects on neuromuscular disorders (NMDs). As a promising molecular therapeutic target against NMDs, AMPK activation by physiological stimulation such as exercise-based training can be beneficial for the treatment of NMDs including Duchenne muscular dystrophy (DMD), spinal muscular atrophy (SMA) and myotonic dystrophy type 1 (DM1) as follows.

Duchenne muscular dystrophy (DMD) is an X-linked recessive neuromuscular dystrophy whose onset is in early childhood primarily affects boys with a symptom of progressive weakness and degeneration of muscle. The dystrophin gene is responsible for DMD and its mutation results in loss of functional dystrophin protein leading to devastation of myofibers and therefore wasting and death of muscle (Bushby et al., 2010; Pilgram et al., 2010; Dial et al., 2018). Glucocorticoides (GCs) and gene therapy with utrophin, a dystrophin homolog are primarily adopted for the treatment of DMD (Bushby et al., 2010; Lim et al., 2017; McDonald et al., 2017; Dial et al., 2018). Copious muscle fibers with slow and oxidative properties are recognized to be more protective against DMD and corrective autophagy signaling alleviates dystrophic pathology (Webster et al., 1988; Sandri et al., 2013; Bujak et al., 2015; Dial et al., 2018). AMPK activation is able to stimulate slow and oxidative phenotypic transition of myofibers and enhanced mitochondrial biogenesis as seen in endurance-based exercise. In addition, AMPK can directly regulate ULK-1 (unc-51-like autophagy activating kinase 1), a master regulator of autophagy, thus initiating autophagic cascades (Vainshtein and Hood, 2016). Collective evidence indicates that AMPK efficiently improves DMD pathology.

Spinal muscular atrophy (SMA) is an autosomal recessive neuromuscular disorder characterized by damage to motor neurons and progressive muscle wasting that often results in early death. Homozygous mutations in SMN1 (survival of motor neuron 1) are responsible for SMA; therefore, SMA patients depend on SMN2 (survival of motor neuron 2), which has a single base difference from SMN1 and mostly produces a truncated nonfunctional product of SMN protein $(\mathrm{SMN} \Delta 7)$ by alternative splicing although a small percentage (10-20\%) of functional full-length SMN2 transcripts are also produced. In addition to deterioration of alpha motor neuron $(\mathrm{aMN})$, aberrant functioning in skeletal muscle itself by abnormal proteins such as SMN $\Delta 7$ appears to also be part of the SMA etiology (Lefebvre et al., 1995; Goulet et al., 2013; Hamilton and Gillingwater, 2013; Dial et al., 2018). AMPK has been suggested as a promising therapeutic target for SMA since it is likely to control transcriptional factors such as CREB or elk-1 (E26 transformation-specific ETS domain containing protein) to upregulate functional full-length SMN (Biondi et al., 2008; Ahmad et al., 2016; Dial et al., 2018). In addition to AMPK being a positive arbitrator of synaptic remodeling, it also appears to rescue synaptic dysfunction of neuromuscular junction (NMJ) in SMA and attenuate mitochondrial dysfunction as an etiology of SMA through augmented functional activities such as mitochondrial biogenesis (d'Ydewalle and Sumner, 2015; Christie-Brown et al., 2017; Scoto et al., 2017; Dial et al., 2018). The SMN-dependent or independent role of AMPK to treat SMA is still under investigation.

Myotonic dystrophy type 1 (DM1) is a dominant autosomal disorder and one of the prevailing forms of 


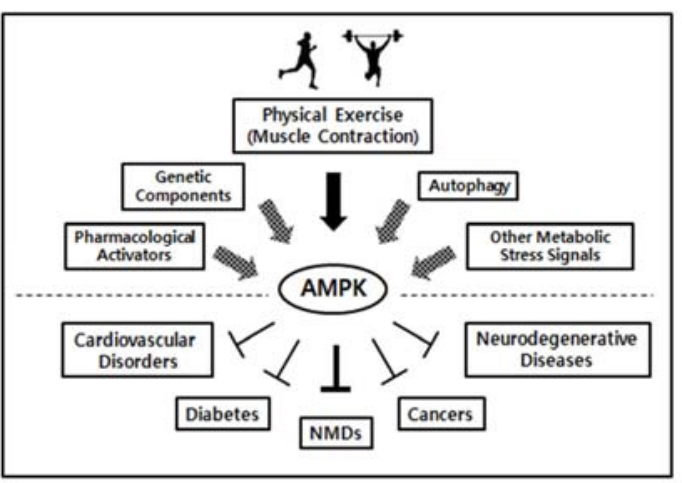

Fig. 3. Potential Impacts of Physical Exercise on Human Health Problems by AMPK Activation. Diverse input components for AMPK activation including physical exercise are designated and a range of health problems including neuromuscular disorders (NMDs) influenced by AMPK signaling are presented.

muscular dystrophy in adults caused by the expansion of CTG trinucleotide repeats in the 3' untranslated region of the DMPK (dystrophia myotonica protein kinase) gene producing abnormal gain-of-function (Cho and Tapscott, 2007; Chau and Kalsotra, 2015). This condition includes functional deregulation of several RNA-binding proteins (RBPs) including MBNL1 (Muscleblind-like 1) and CELF1 (CUG-BP Elav-like family member 1) in addition to the alternative splicing of $\mathrm{ClC}-1$ (skeletal muscle-specific chloride channel) transcripts whose normal function contributes to optimal control of excitability and contractile activity in skeletal muscle (Cho and Tapscott, 2007; Chau and Kalsotra, 2015). It has also been suggested that AMPK activation by exercise or pharmacological agonists with specificity is likely to rescue abnormal splicing patterns along with corrective autophagy signaling to remove dysfunctional proteins in DM1. As a downstream target of AMPK, PGC-1a is also believed to contribute to AMPK signaling in NMDs and to regulation of mRNA processing to correct and mitigate DM1 myopathy (Dial et al., 2018).

Collectively, many studies robustly support AMPK as a favorable therapeutic target for various neuromuscular disorders. Although not described in detail in this review, plenty of evidence also strongly supports that the therapeutic potential of AMPK signaling can be helpful for the treatment of other human health problems such as cardiovascular disorders including hypertension, diabetes, neurodegenerative diseases and certain types of cancers as well (Richter and Ruderman, 2009) (Fig. 3).

\section{Conclusion and Future Perspectives}

Recent collective evidence has proposed the relevance of AMPK as a favorable molecular target in neuromuscular systems including skeletal muscle and NMDs. AMPK is a key interpreter that explains the phenotypic adaptive responses in single or combined modes of exercise. Successive efforts to identify the best conditions of each exercise training program (ET, RT or CT) might also be important and helpful to maximize therapeutic outcomes against diverse health problems including NMDs. In that sense, AMPK and AMPK-related signaling can be a fundamentally practical check point to evaluate and determine the effectiveness of therapeutic potential at the molecular level of verification. Moreover, combinations of individual single modes of exercise training (ET or RT), if desired, should be considered in terms of sequence, intensity and duration based on the pros and cons of ET and RT for optimization.

Although many bits of information regarding the function of AMPK in various exercise-based training procedures has yet to be clearly defined and revealed, as a lifestyle-based tactic, it is worth to keep pinpointing its precise role in those procedures. AMPK is likely to be a promising therapeutic target for the prevention, amelioration or cure of many health problems including NMDs. The potential impact of AMPK needs to be further revealed and novel therapeutic interventions should be established. Particularly, as a therapeutic regimen, exercise-based training reflecting AMPK signaling biology 
might also be beneficial for physical rehabilitation and even for emergency situations in which acute metabolic stresses are elicited.

\section{Acknowledgements}

This work was supported by the National Research Foundation of Korea (NRF-2017R1D1A1B03028729).

\section{References}

Ahmad S, Bhatia K, Kannan A, et al. Molecular mechanisms of neurodegeneration in spinal muscular atrophy. $\mathrm{J}$ Exp Neurosci. 2016;10:39-49.

Baar K. Training for endurance and strength: lessons from cell signaling. Med Sci Sports Exerc. 2006;38(11): 1939-44.

Bassel-Duby R, Olson EN. Signaling pathways in skeletal muscle remodeling. Annu Rev Biochem. 2006;75: 19-37.

Beyfuss K, Hood DA. A systematic review of p53 regulation of oxidative stress in skeletal muscle. Redox Rep. 2018;23(1):100-17.

Biondi O, Grondard C, Lecolle S, et al. Exercise-induced activation of NMDA receptor promotes motor unit development and survival in a type 2 spinal muscular atrophy model mouse. J Neurosci. 2008;28(4):953-62.

Bujak AL, Crane JD, Lally JS, et al. AMPK activation of muscle autophagy prevents fasting-induced hypoglycemia and myopathy during aging. Cell Metab. 2015;21(6):883-90.

Bushby K, Finkel R, Birnkrant DJ, et al. Diagnosis and management of Duchenne muscular dystrophy, part 1: diagnosis, and pharmacological and psychosocial management. Lancet Neurol. 2010;9(1):77-93.

Camera DM, Smiles WJ, Hawley JA. Exercise-induced skeletal muscle signaling pathways and human athletic performance. Free Radic Biol Med. 2016;98:131-43.
Cao S, Li B, Yi X, et al. Effects of exercise on AMPK signaling and downstream components to PI $3 \mathrm{~K}$ in rat with type 2 diabetes. PLoS One. 2012;7(12):e51709.

Chau A, Kalsotra A. Developmental insights into the pathology of and therapeutic strategies for DM1: Back to the basics. Dev Dyn. 2015;244(3):377-90.

Cho DH, Tapscott SJ. Myotonic dystrophy: emerging mechanisms for DM1 and DM2. Biochim Biophys Acta. 2007;1772(2):195-204.

Christie-Brown V, Mitchell J, Talbot K. The SMA Trust: the role of a disease-focused research charity in developing treatments for SMA. Gene Ther. 2017; 24(9):544-6

Chtara M, Chaouachi A, Levin GT, et al. Effect of concurrent endurance and circuit resistance training sequence on muscular strength and power development. J Strength Cond Res. 2008;22(4):1037-45.

Coffey VG, Hawley JA. The molecular bases of training adaptation. Sports Med. 2007;37(9):737-63.

Coffey VG,Hawley JA. Concurrent exercise training: do opposites distract? J Physiol. 2017;595(9):2883-96.

Deshmukh AS, Hawley JA, Zierath JR. Exercise-induced phospho-proteins in skeletal muscle. Int J Obes (Lond). 2008;32(Suppl 4):S18-23.

Dial AG, Ng SY, Manta A, et al. The Role of AMPK in Neuromuscular Biology and Disease. Trends Endocrinol Metab. 2018;29(5):300-12.

Dudley GA, Djamil R. Incompatibility of endurance- and strength-training modes of exercise. J Appl Physiol (1985). 1985;59(5):1446-51.

d'Ydewalle C, Sumner CJ. Spinal Muscular Atrophy Therapeutics: Where do we Stand? Neurotherapeutics. 2015;12(2):303-16

Fry AC. The role of resistance exercise intensity on muscle fibre adaptations. Sports Med. 2004;34(10):663-79.

Gibala M. Molecular responses to high-intensity interval exercise. Appl Physiol Nutr Metab. 2009;34(3): 428-32. 
Gomes AS, Ramos H, Soares J, et al. p53 and glucose metabolism: an orchestra to be directed in cancer therapy. Pharmacol Res. 2018;131:75-86.

Goulet BB, Kothary R, Parks RJ. At the "junction" of spinal muscular atrophy pathogenesis: the role of neuromuscular junction dysfunction in SMA disease progression. Curr Mol Med. 2013;13(7):1160-74.

Gowans GJ, Hawley SA, Ross FA, et al. AMP is a true physiological regulator of AMP-activated protein kinase by both allosteric activation and enhancing net phosphorylation. Cell Metab. 2013;18(4):556-66.

Hamilton G, Gillingwater TH. Spinal muscular atrophy: going beyond the motor neuron. Trends Mol Med. 2013;19(1):40-50.

Hardie DG. AMP-activated protein kinase: a cellular energy sensor with a key role in metabolic disorders and in cancer. Biochem Soc Trans. 2011a;39(1):1-13.

Hardie DG. Sensing of energy and nutrients by AMP-activated protein kinase. Am J Clin Nutr. 2011b;93(4):891S-6.

Hawley JA, Hargreaves M, Joyner MJ, et al. Integrative biology of exercise. Cell. 2014;159(4):738-49.

Hawley JA, Hargreaves M, Zierath JR. Signalling mechanisms in skeletal muscle: role in substrate selection and muscle adaptation. Essays Biochem. 2006;42:1-12.

Hawley JA. Molecular responses to strength and endurance training: are they incompatible? Appl Physiol Nutr Metab. 2009;34(3):355-61.

Hickson RC. Interference of strength development by simultaneously training for strength and endurance. Eur J Appl Physiol Occup Physiol. 1980;45(2-3): 255-63.

Holloszy JO, Coyle EF. Adaptations of skeletal muscle to endurance exercise and their metabolic consequences. J Appl Physiol Respir Environ Exerc Physiol. 1984;56(4):831-8.

Holloszy JO. Biochemical adaptations in muscle. Effects of exercise on mitochondrial oxygen uptake and respiratory enzyme activity in skeletal muscle. J Biol
Chem. 1967;242(9):2278-82.

Jensen TE, Rose AJ, Jorgensen SB, et al. Possible CaMKKdependent regulation of AMPK phosphorylation and glucose uptake at the onset of mild tetanic skeletal muscle contraction. Am J Physiol Endocrinol Metab. 2007;292(5):1308-17.

Jensen TE, Wojtaszewski JF, Richter EA. AMP-activated protein kinase in contraction regulation of skeletal muscle metabolism: necessary and/or sufficient? Acta Physiol (Oxf). 2009;196(1):155-74.

Jones TW, Howatson G, Russell M, et al. Performance and neuromuscular adaptations following differing ratios of concurrent strength and endurance training. J Strength Cond Res. 2013;27(12):3342-51.

Kjobsted R, Hingst JR, Fentz J, et al. AMPK in skeletal muscle function and metabolism. FASEB J. 2018;32(4): 1741-77.

Kwon I, Jang Y, Cho JY, et al. Long-term resistance exercise-induced muscular hypertrophy is associated with autophagy modulation in rats. J Physiol Sci. 2018;68(3):269-80.

Lefebvre S, Burglen L, Reboullet S, et al. Identification and characterization of a spinal muscular atrophydetermining gene. Cell. 1995;80(1):155-65.

Lim KR, Maruyama R, Yokota T. Eteplirsen in the treatment of Duchenne muscular dystrophy. Drug Des Devel Ther. 2017;11:533-45.

McDonald CM, Campbell C, Torricelli RE, et al. Ataluren in patients with nonsense mutation Duchenne muscular dystrophy (ACT DMD): a multicentre, randomised, double-blind, placebo-controlled, phase 3 trial. Lancet. 2017;390(10101):1489-98.

Morales-Alamo D, Calbet JAL. AMPK signaling in skeletal muscle during exercise: Role of reactive oxygen and nitrogen species. Free Radic Biol Med. 2016;98:68-77.

Mounier R, Theret M, Lantier L, et al. Expanding roles for AMPK in skeletal muscle plasticity. Trends Endocrinol Metab. 2015;26(6):275-86. 
Murach KA, Bagley JR. Skeletal Muscle Hypertrophy with Concurrent Exercise Training: Contrary Evidence for an Interference Effect. Sports Med. 2016;46(8): 1029-39.

Murlasits Z, Kneffel Z, Thalib L. The physiological effects of concurrent strength and endurance training sequence: A systematic review and meta-analysis. J Sports Sci. 2018;36(11):1212-9.

ONeill HM. AMPK and Exercise: Glucose Uptake and Insulin Sensitivity. Diabetes Metab J. 2013;37(1):1-21.

Perez-Schindler J, Hamilton DL, Moore DR, et al. Nutritional strategies to support concurrent training. Eur J Sport Sci. 2015;15(1):41-52.

Pilgram GS, Potikanond S, Baines RA, et al. The roles of the dystrophin-associated glycoprotein complex at the synapse. Mol Neurobiol. 2010;41(1):1-21.

Preston RR, Wilson TE. Physiology. Wolters Kluwer Health/Lippincott Williams \& Wilkins. 2013. Reidy PT, Konopka AR, Hinkley JM, et al. The effect of feeding during recovery from aerobic exercise on skeletal muscle intracellular signaling. Int J Sport Nutr Exerc Metab. 2014;24(1):70-8.

Richter EA, Ruderman NB. AMPK and the biochemistry of exercise: implications for human health and disease. Biochem J. 2009;418(2):261-75.

Sakamoto K, Goodyear LJ. Invited review: intracellular signaling in contracting skeletal muscle. J Appl Physiol (1985). 2002;93(1):369-83.

Sandri M, Coletto L, Grumati P, et al. Misregulation of autophagy and protein degradation systems in myopathies and muscular dystrophies. J Cell Sci. 2013;126(23): 5325-33.

Scoto M, Finkel RS, Mercuri E, et al. Therapeutic approaches for spinal muscular atrophy (SMA). Gene Ther. 2017;24(9):514-9.
Stein SC, Woods A, Jones NA, et al. The regulation of AMP-activated protein kinase by phosphorylation. Biochem J. 2000;345(3):437-43.

Tesch PA. Skeletal muscle adaptations consequent to long-term heavy resistance exercise. Med Sci Sports Exerc. 1988;20(5 Suppl):132-4.

Vainshtein A, Hood DA. The regulation of autophagy during exercise in skeletal muscle. J Appl Physiol (1985). 2016;120(6):664-73.

Vander Heiden MG, Cantley LC, Thompson CB. Understanding the Warburg effect: the metabolic requirements of cell proliferation. Science. 2009;324(5930):1029-33.

Vousden KH, Ryan KM. p53 and metabolism. Nat Rev Cancer. 2009;9(10):691-700.

Wang L, Mascher H, Psilander N, et al. Resistance exercise enhances the molecular signaling of mitochondrial biogenesis induced by endurance exercise in human skeletal muscle. J Appl Physiol (1985). 2011;111(5): 1335-44.

Warburg O. Notiz uber den Stoffwechsel der Tumoren. Biochem. Zeitschr. 1930;228 (1/3):257-8.

Warburg O. On the origin of cancer cells. Science. 1956;123(3191):309-14.

Webster C, Silberstein L, Hays AP, et al. Fast muscle fibers are preferentially affected in Duchenne muscular dystrophy. Cell. 1988;52(4):503-13.

Widegren U, Ryder JW, Zierath JR. Mitogen-activated protein kinase signal transduction in skeletal muscle: effects of exercise and muscle contraction. Acta Physiol Scand. 2001;172(3):227-38.

Wilson JM, Marin PJ, Rhea MR, et al. Concurrent training: a meta-analysis examining interference of aerobic and resistance exercises. J Strength Cond Res. 2012;26(8):2293-307. 